\title{
The Generalized Integro-Exponential Function
}

\author{
By M. S. Milgram
}

\begin{abstract}
The generalized integro-exponential function is defined in terms of the exponential integral (incomplete gamma function) and its derivatives with respect to order. A compendium of analytic results is given in one section. Rational minimax approximations sufficient to permit the computation of the first six first-order functions are reported in another section.
\end{abstract}

1. Introduction. The generalized integro-exponential function plays an important role in the theory of transport processes and fluid flow, yet no unified summary of its properties exists in the literature. First introduced by Van de Hulst [12] who gave a power series and numerical tabulation for some special cases, the function was abstracted by Chandrasekhar [8] and recently reviewed by Van de Hulst [13]. In the interim, tabulated values were presented by Stankiewicz [26] and Gussmann [11]; the latter as well as Abu-Shumays [2] and Kaplan [16] independently summarized some analytic properties. Recent work by this author [20]-[22] has reasserted the significant role played by this function in transport theory, and, by invoking the theory of Meijer's $G$-functions [18], many general properties of this function have been unveiled which were previously unknown. These were summarized in an Appendix to [20] and in a Table in a conference proceedings [22].

Since these summaries appeared, the problem of accurately evaluating this function numerically has arisen. For small values of the real independent variable $x$, power series evaluations work reasonably well with coefficients obtainable from the formulas given in [20] and [22]. For intermediate values of $x$, power series break down due to cancellation of significant digits when evaluating differences between large numbers, and for large values of $x$, asymptotic series do not do justice to the inherent accuracy available with modern computers.

The purpose of this paper is then twofold:

(1) Reiterate, derive and present a unified summary of the analytic properties of these functions only some of which can be found elsewhere;

(2) Proffer some approximations on which the numerical evaluation of a large number of these functions can be based.

2. Analysis. Let $s$ and $z$ be continuous (complex) variables and $n$ and $j$ represent nonnegative integers. Define the "generalized integro-exponential function" $E_{s}^{j}(z)$ by

$$
E_{s}^{j}(z)=\frac{(-1)^{j}}{j !} \frac{\partial^{j}}{\partial s^{j}} E_{s}(z)
$$

Received December 1, 1981; revised April 30, 1982, October 25, 1982, November 28, 1983, February 17, 1984, and March 16, 1984.

1980 Mathematics Subject Classification. Primary 33A70, 41A20, 85A25.

1985 American Mathematical Society $0025-5718 / 85 \$ 1.00+\$ .25$ per page 
where $E_{s}(z)$ is the usual* exponential integral generalized to $n=s$, or in terms of incomplete gamma functions

$$
E_{s}(z)=z^{s-1} \Gamma(1-s, z) .
$$

We have the integral representation

$$
E_{s}^{0}(z) \equiv E_{s}(z)=\int_{1}^{\infty} t^{-s} \exp (-z t) d t
$$

for the case $s=n$ this reduces to the exponential integral about which a considerable literature revolves [1], [3], [4], [6], [9], [14], [19], [23], [25], [27], [29]. Apply Eq. (2.1) to (2.2), interchange the order of integration and differentiation, and obtain the integral representation

$$
E_{s}^{j}(z)=\frac{1}{\Gamma(j+1)} \int_{1}^{\infty}(\log t)^{j} t^{-s} \exp (-z t) d t
$$

which, when $s=n$, reduces to Gussmann's [11] definition $E_{n, j}(z)=E_{n}^{j}(z)$, or that of van de Hulst [12] $E_{1}^{(j)}(z)=E_{1}^{j-1}(z)$. Equation (2.3) may be integrated by parts (integrate $t^{-s}$ and differentiate the remaining factors) to obtain the recursion formula

$$
E_{s}^{j}(z)=\left(z E_{s-1}^{j}(z)-E_{s}^{j-1}(z)\right) /(1-s), \quad s \neq 1, j \geqslant 0,
$$

defining $E_{s}^{-1}(z)=\exp (-z)$ for the case $j=0$.

To obtain a unified theory of this function and its properties, start with the known identification of $\exp (-z)$ in terms of Meijer's $G$-function [18, No. 6.2.1(1) and 6.4(1)]

$$
\exp (-z)=G_{0,1}^{1,0}\left(z \mid \begin{array}{c}
; \\
0 ;
\end{array}\right)=\frac{1}{2 \pi i} \int_{L_{0}} \Gamma(-t) z^{t} d t
$$

substitute into Eq. (2.2), and use a known [18, No. 5.6.4(6)] integration formula to obtain

$$
\begin{aligned}
E_{s}(z) & =G_{1.2}^{2,0}\left(z \mid \begin{array}{l}
; s \\
0, s-1 ;
\end{array}\right)=\frac{1}{2 \pi i} \int_{L_{0}} \frac{\Gamma(-t) z^{t}}{s-1-t} d t \\
& =\exp (-z) z^{s-1} \psi(s, s ; z),
\end{aligned}
$$

where $L_{0}$ is the negatively directed contour enclosing both the nonnegative $t$-axis, and the pole at $t=s-1$. The third term in the equality (2.6a) is the contour integral representation [18, No. 5.2(1)] of the $G$-function according to prescription. In Eq. (2.6b), $\psi$ is a confluent hypergeometric function of the second kind. Operating on Eq. (2.6a) as in Eq. (2.1), we obtain the contour integral representation

$$
\begin{aligned}
& E_{s}^{j}(z)=\frac{1}{2 \pi i} \int_{L_{0}} \frac{\Gamma(-t) z^{t}}{(s-1-t)^{j+1}} d t \\
& =G_{j+1, j+2}^{j+2,0}\left(z \mid \begin{array}{l}
; s, \ldots, s \\
0, s-1, \ldots, s-1 ;
\end{array}\right) .
\end{aligned}
$$

* "Exponential integral" (of order $s$ ) refers to the function $E_{s}(z)$. Some authors [13], [1] use this nomenclature to mean $E_{n}(z)$, while others [18] mean $E_{1}(z)$, or $\operatorname{Ei}(z)=-E_{1}(-z)$ or both. 
The result $(2.7 \mathrm{~b})$ is obtained by identifying the contour integral (2.7a) according to prescription [18, No. 5.2(1)]; Eq. (2.7a) provides a means of generalizing the function to noninteger values of $j$. Set $j=-1$ in Eq. (2.7a), and with reference to Eq. (2.5) obtain

$$
E_{s}^{-1}(z)=\exp (-z)
$$

from which it is easy to see how Eq. (2.4) reduces to a well-known result $[1$, No. 5.1.14] when $j=0$ and $s=n$.

The power series follows immediately from Eq. (2.7a) by evaluating the residues of the simple poles of $\Gamma(-t)$ and the multi-poles of order $j+1$ when $s \neq n$ or $j+2$ if $s=n$. The results are

$$
\begin{aligned}
E_{s}^{j}(z)= & \sum_{l=0}^{\infty} \frac{(-z)^{l}}{(s-1-l)^{j+1} l !} \\
& +\frac{z^{s-1}(-1)^{j}}{j !} \sum_{l=0}^{j}(-1)^{l}\left(\begin{array}{l}
j \\
l
\end{array}\right) \log ^{j-l} z \Gamma(1-s)^{(l)}, \quad s \neq n,
\end{aligned}
$$

and

$$
\begin{aligned}
E_{n}^{j}(z)= & \sum_{\substack{l=0 \\
l \neq n-1}}^{\infty}, \frac{(-z)^{l}}{(n-1-l)^{j+1} l !} \\
& +\frac{z^{n-1}(-1)^{j+n}}{(j+1) !} \sum_{l=0}^{j+1}\left(\begin{array}{c}
j+1 \\
l
\end{array}\right) \log ^{1+j-l} z \Psi_{l, n},
\end{aligned}
$$

where $\Gamma(1-s)^{(l)}$ means the $l$ th derivative of $\Gamma(1-s)$ with respect to $s$, and

$$
\Psi_{l, n}=\lim _{t \rightarrow n-1} \frac{\partial^{\prime}}{\partial t^{\prime}}\left(\frac{\Gamma(t-n+2) \Gamma(n-t)}{\Gamma(1+t)}\right) .
$$

Analytic expressions for $\Psi_{l, n}(0 \leqslant l \leqslant 8)$ have been obtained through the use of computer algebra [28]; they are given in Table 1. General, but complicated expressions can be found in [2, Eq. (4.12)]. The result (2.10) is also given in [11] and [2, Eq. (4.7)]. Similar coefficients may be obtained for the functions $E_{-n}^{j}(z)$; however an alternative method for the evaluation of the latter set of functions will be given shortly.

It is of some interest to obtain other integral representations. From Eq. (2.7b) we recognize the $G$-function as an Euler transform [18, No. 5.6.4(6)]:

$$
E_{s}^{j}(z)=\int_{1}^{\infty} t^{-s} E_{s}^{j-1}(z t) d t, \quad j \geqslant 0,
$$

from which the name derives. The case $j=1, s=1$ has been obtained elsewhere [17] in the form of a power series; (2.12) may also be found in [2]. An alternate expression for Eq. (2.12) is the repeated integral form:

$$
E_{s}^{j}(z)=\int_{1}^{\infty} d y_{1} y_{1}^{-s} \int_{1}^{\infty} d y_{2} \cdots \int_{1}^{\infty} d y_{j+1} y_{j+1}^{-s} \exp \left(-z \prod_{i=1}^{j+1} y_{i}\right) .
$$

From (2.2), (2.3) and (2.12), we find

$$
E_{s}^{j}(z)=\frac{1}{\Gamma(j)} \int_{1}^{\infty}(\log t)^{j-1} t^{-s} E_{s}(z t) d t, \quad j>0,
$$


from which it may be shown that

$$
\begin{aligned}
\int_{1}^{\infty} E_{s}^{l}(z t) t^{-s} \log ^{k} t d t \\
\quad=\frac{\Gamma(k+1)}{\Gamma(l)} \int_{1}^{\infty} E_{s}^{k+1}(z t) t^{-s} \log ^{l-1} t d t, \quad l>0, k \geqslant 0 .
\end{aligned}
$$

Finally, using (2.14a) and (2.14b) we find the remarkable representation

$$
E_{s}^{j}(z)=\frac{1}{\Gamma(j-l)} \int_{1}^{\infty} E_{s}^{\prime}(z t) t^{-s} \log ^{j-l-1} t d t \quad \forall 0 \leqslant l \leqslant j-1
$$

by a process of induction, employing (2.19). This generalizes both (2.12) and (2.14a).

Starting from Frullani's integral

$$
\log t=\int_{0}^{\infty} d v(\exp (-v)-\exp (-t v)) / v
$$

and Eq. (2.3), the order of integration may, with care, be interchanged, giving the Laplace transform

$$
E_{s}^{1}(z)=\int_{0}^{\infty} d v \exp (-v)\left[E_{s}(z)-\exp (v) E_{s}(z+v)\right] / v .
$$

After applying Eq. (2.1) to Eq. (2.15), a more general form is found:

$$
\begin{array}{r}
E_{s}^{j+1}(z)=\frac{1}{(j+1)} \int_{0}^{\infty} d v \exp (-v)\left[E_{s}^{j}(z)-\exp (v) E_{s}^{j}(z+v)\right] / v, \\
j \geqslant 0 .
\end{array}
$$

A number of $G$-function representations may also be derived. From Eq. (2.2) and known integration formula [18, No. 5.6.2(18)], we have

$$
E_{s}(z)=\exp (-z) G_{1,2}^{2,1}\left(z \mid \begin{array}{l}
0 \\
0, s-1 ;
\end{array}\right) / \Gamma(s) .
$$

Starting with Eq. (2.7a), substitute $t:=2 t$ and use the duplication formula for the gamma function to find [18, No. 5.2(1)]

$$
E_{s}^{j}(z)=\frac{2^{-j-1}}{\sqrt{\pi}} G_{j+1, j+3}^{j+3,0}\left(\begin{array}{l|l}
\frac{z^{2}}{4} \mid \begin{array}{l}
;(s+1) / 2, \ldots,(s+1) / 2 \\
0, \frac{1}{2},(s-1) / 2, \ldots,(s-1) / 2 ;
\end{array}
\end{array}\right) .
$$

From Eq. (2.3) the differentiation rules are easy to uncover

$$
\begin{gathered}
\frac{\partial^{k}}{\partial z^{k}} E_{s}^{j}(z)=(-1)^{k} E_{s-k}^{j}(z), \\
\frac{\partial^{m}}{\partial s^{m}} E_{s}^{j}(z)=\frac{(-1)^{m} \Gamma(m+j+1)}{\Gamma(j+1)} E_{s}^{m+j}(z),
\end{gathered}
$$

and from Eqs. (2.19) and (2.4) we discover that $E_{s}^{j}(z)$ satisfies

$$
(s-1) \frac{\partial}{\partial s} E_{s}^{j}(z)-z \frac{\partial^{2}}{\partial s \partial z} E_{s}^{j}(z)+(j+1) E_{s}^{j}(z)=0
$$

as well as [18, No. 5.8(1)]

$$
\left(1+\frac{\partial}{\partial z}\right)\left(z \frac{\partial}{\partial z}-s+1\right)^{j+1} E_{s}^{j}(z)=0 .
$$


A short table of integrals is included as Appendix A.

In applications [27] we need to evaluate $E_{-n}^{j}(x), n>0, x=\operatorname{Re}(z)>0$. Integrating by parts in Eq. (2.3) leads to the result

$$
E_{0}^{j}(z)=E_{1}^{j-1}(z) / z, \quad j \geqslant 0 .
$$

Repeated application of Eq. (2.4) gives

$$
E_{-n}^{j}(z)=\frac{\Gamma(n+1)}{z^{n}}\left(\sum_{k=0}^{n-1} \frac{z^{k} E_{-k}^{j-1}(z)}{\Gamma(k+2)}+E_{0}^{j}(z)\right) .
$$

Similarly, repeated application of Eq. (2.21) leads to the simpler form

$$
E_{-n}^{j}(z)=\frac{\Gamma(n+1)}{z^{n+1}}\left[\exp (-z) \sum_{l=0}^{n-j} \frac{z^{l}}{l !} \xi_{l, n}^{j}+\sum_{m=1}^{j} \xi_{0, n}^{m-1} E_{1}^{j-m}(z)\right],
$$

where the (precomputed) constants $\xi_{l, n}^{j}$ are given analytically by

$$
\begin{aligned}
& \xi_{l, n}^{j}=\sum_{k_{j}=0}^{n-j-l} \sum_{k_{j-1}=0}^{k_{j}} \cdots \sum_{k_{1}=0}^{k_{2}}\left(\frac{1}{k_{j}+l+j}\right)\left(\frac{1}{k_{j-1}+l+j-1}\right) \cdots\left(\frac{1}{k_{1}+l+1}\right), \\
& \xi_{n-j, n}^{j}=\frac{(n-j) !}{n !}, \quad \xi_{l, n}^{0}=1, \quad \xi_{l, n}^{j}=0 \quad \text { if } n<j+l .
\end{aligned}
$$

The derivation of Eq. (2.22) comes by proving its truth for the cases $j=1$ and $j=2$ and proceeding by induction, using the identities

$$
\begin{gathered}
\xi_{0, n}^{j}=\sum_{k=0}^{n-1}\left(\frac{1}{k+1}\right) \xi_{0, k}^{j-1}, \\
\xi_{l, n}^{j}=\sum_{k=0}^{n-l-j}\left(\frac{1}{k+l+j}\right) \xi_{l, k+l+j-1}^{j-1} .
\end{gathered}
$$

From Eq. (2.22) all the $E_{-n}^{j}(z)$ can be obtained for $n \geqslant 0$ if the functions $E_{1}^{m}(z)$ are known for $0 \leqslant m \leqslant j-1$. Approximations for $E_{1}^{m}(z)$ are given in Section 3. A lengthy form for the evaluation of $E_{n}^{j}(x)$ for $n>1$ in terms of $E_{1}^{j}(x)$ is given by Gussmann [11, Eq. (A.18) to (A.21')]. Note that Eq. (2.22) is numerically stable since all its terms are positive.

For large values of real $z$, the first few terms in the asymptotic series of $E_{-n}^{j}(x)$ are given by the first finite sum in Eq. (2.22). From Eq. (2.7b) and the theory [18, Section 5.10] of $G$-functions, the asymptotic series is theoretically known. The first terms are

$$
E_{s}^{j}(z) \sim \exp (-z) z^{-(j+1)}[1-(j+1)(j+2 s) /(2 z)+\cdots]
$$

A more general result can be obtained by integrating Eq. (2.12) repeatedly by parts:

$$
E_{n}^{j+1}(z)=\frac{1}{z} \sum_{k=0}^{\infty} \frac{(-1)^{k}}{z^{k}} \frac{\Gamma(n+k)}{\Gamma(n)} E_{n+k+1}^{j}(z) .
$$

The claimed asymptotic formula is then $(\operatorname{Re}(z) \rightarrow+\infty)$

$$
E_{n}^{j}(z) \sim \exp (-z) z^{-j-1} \sum_{l=0}^{P} \frac{(-1)^{l}}{z^{l}} \frac{\Gamma(n+l+j)}{\Gamma(n)} \xi_{n-1, n+l+j-1}^{j},
$$


where $P$ is an integer. This can be proven by establishing its truth for the case $j=0$ and $j=1$, using in Eq. (2.26) the well-known [1, Eq. 5.1.51] result

$$
E_{n+k+1}(z) \sim \exp (-z) z^{-1} \sum_{m=0}^{P} \frac{(-1)^{m}}{z^{m}} \frac{\Gamma(n+k+m+1)}{\Gamma(n+k+1)},
$$

and proceeding by induction, employing the identity

$$
\sum_{k=0}^{l} \frac{1}{(n+k)} \xi_{n+k, n+l+j}^{j}=\xi_{n-1, n+l+j}^{j+1}
$$

in Eq. (2.27).

Finally, from Eq. (2.9) we have the special value

$$
E_{s}^{j}(0)=\left(\frac{1}{s-1}\right)^{j+1}, \quad \operatorname{Re}(s)>1
$$

3. Rational Minimax Approximations to $E_{1}^{j}(x)$. With the aid of the computer code REMES2 [15], it proved possible to obtain rational minimax approximations to $E_{1}^{j}(x)$ for $x$ real, $1 \leqslant j \leqslant 6$, and hence $E_{-n}^{j}(x)$ with $1 \leqslant j \leqslant 7, n>0$ because of Eq. (2.22). The region $0 \leqslant x$ was conveniently divided into three ranges for the purpose of both evaluating the master functions and obtaining efficient fits.

3a. Small $x$. In this range, we evaluate $E_{1}^{j}(x)$ according to the power series (2.10) in the form

$$
E_{1}^{j}(x)=P_{k}(x) / Q_{l}(x)+P_{1 j}(\log x),
$$

where $P_{k}(x) / Q_{l}(x)$ is the $(k, l)$ minimax fit to the infinite series plus the constant $(l=j+1)$ term of the second sum in Eq. (2.10). $P_{1 j}(\log x)$ is a polynomial in $\log \left(e^{\gamma} x\right)$ of order $j$ obtainable from Table 1 by means of computer algebra. Although no proof has been found, for $1 \leqslant j \leqslant 7$, this polynomial $P_{1 j}(\log x)$ can be written

$$
P_{1 j}=\sum_{l=1}^{j+1} u_{j, l} v^{l}
$$

where

$$
v=\log \left(e^{\gamma} x\right)
$$

and

$$
u_{j, l}=-u_{j-1, l-1} / l, \quad l \geqslant 1,
$$

with the constants $u_{j, 0}$ given in Table 2, and $\gamma$ being Euler's constant. The upper end of this range $\left(X_{U}\right)$ was chosen to coincide with a point smaller than the first positive zero of $E_{1}^{j}(x)-P_{1 j}(\log x)$. All master function calculations were done in 120 bit double-precision arithmetic ( $\sim 28$ s.d.) and the approximation chosen was the lowest $(k+l)$ entry in the Walsh array with more than 14 s.d. accuracy. The coefficients of the $(k, l)$ minimax fits are given in Table 3.

$3 b$. Intermediate $x$. In this range, $X_{U} \leqslant x \leqslant 10$, the master functions were obtained from the power series (2.10), with an exponential weighting such that

$$
E_{1}^{j}(x)=\exp (-x) P_{k}(x) / Q_{l}(x)
$$


TABLE 1

Analytic expressions for $\Psi_{l, n}$ in Eq. (2.10)

$\operatorname{CPSI}(1)=$ GAMIN $\$ \quad \operatorname{CPSI}(2)=$ GAMIN*(-PSIN)

CPSI $(\Xi)=+$ GAMIN* (PSIN**2-PSIN1+E. *ZETAZ)

$\operatorname{CPSI}(4)=+$ GAMIN* (3. *PSIN*PSIN1-E. *PSIN*ZETAE-PSIN**3-PSINE)

$\operatorname{CPSI}(5)=$

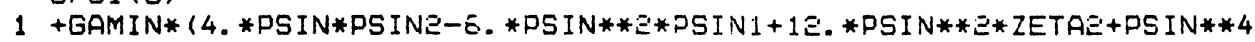

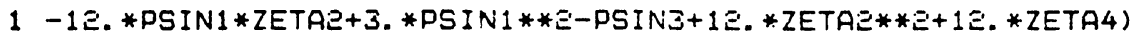

$\operatorname{CPSI}(6)=$

$1+$ GAMIN*160. *PSIN*PSIN1*ZETAZ-15. *PSIN*PSIN1**Z+5. *PSIN*PSIN3-60.

1 *PSIN*ZETA2**Z-6Q. *PSIA*ZSTA4-10.*PSIN**2*PSINE+10. *PSIN**3*PSIN

1 1-ZQ. *PSIN**3*ZETAZ-PSIN**5+10. *PSIN1*PSINE-EQ1. *PSINE*ZETAE-PSIN

$14)$

$\operatorname{CPSI}(7)=$

$1+$ +GAMIN*1-EQ. *PSIN*PSIN : *PSINE+1ZQ. *FSIN*PSINE*ZETAE+E. *PSIN*PSIN

$14-180$. *PSIN**Z*PSIN1*ZETAE+45. *PSIN**Z*PSIN1**Z-15. *PSIN**Z*PSIN

$13+18 Q . * P S I N * * 2 * Z E T A Z * * \Xi+180 . * P S I N * * 2 * Z E T A 4+2 Q . * P S I N * 3 * P S I N E-15$.

1 *PSIN**4*PSIN1+30. *PSIN**4*ZETAE+PSIN**E+15. *PSIN1*PSIN3-18Q. *PS

1 IN1*ZETAZ**Z-180.*PSIN *ZITA4+9Q.*PSIN1**E*ZETAE-15.*PSIN1**3+10

1 .*PSINE**E-3Q.*PSIN3*ZETAE-PSINS+3EQ.*ZETAE*ZETA4+1EQ.*ZETAE**3+

1 240. * ZETAE)

$\operatorname{CPSI}(8)=$

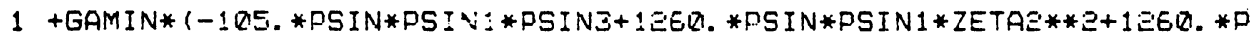

1 SIN*PSIN1*ZETA4-E3Q.*FSIN*PSIN1**2*ZETAE+105. *PSIN*PSIN1**3-7Q.*

1 PSIN*PSINE**Z+210. *PSIN*PSIN3*ZETRE+7. *PSIN*PSINS-252Q. *PSIN*ZET

1 AZ*ZETA4-84Q. *PSIN*ZETAE**3-1680. *PSIN*ZETAE+E10. *PSIN**Z*DSIN1*

1 PSINE-420. *PSIN**2*PSIN三*ZETAE-21. *PSIN**2*PSIN4+4E0. *PSIN**3*PS

1 IN1*ZETAE-1 125. *PSIN**3*DSIN1**2+35.*PSIN**3*PSIN3-4201*PSIN**3*Z

1 ETA2**Z-4ZR. *PSIN**3*ZETA4-35.*PSIN**4*PSINE+E1. *PSIN**5*PSIN1-4

1 E. *PSIN**5*ZETA2-PSIN**7+420. *PSIN1*PSINZ*ZETA2+21. *PSIN1*PSIN4-

1 105. *PSIN1**Z*PSINE+35. *PSINE*PSIN3-4E0. *PSINE*ZETAE**E-420. *PSI

1 NE*ZETA4-4Ë.*PSIN4*ZETRE-PSING)

$\operatorname{CPSI}(9)=$

1 +GAMIN*1-3360. *PSIN*PSIN1*PSINE*ZETAE-168. *PSIN*PSIN1*PSIN4+840.

1 *PSIN*PSIN1**2*PSINE-298. *PSIN*PSINE*PSIN3+3360. *PSIN*PSINE*ZETA

$12 * * 2+3360$. *PSIN*PSIN2*ZETA4+33E. *PSIN*PSIN4*ZETA2+8. *PSIN*PSING+

$1420 . * P S I N * * Z * P S I N 1 * P S I A 3-5 Q 40 . * P S I N * * Z * P S I N 1 * Z E T A Z * * Z-5 Q 40$. *PSIN

1 **2*PSIN1*ZETA4+2SER. *PSIN**2*PSIN1**2*ZETA2-420. *PSIN**2*PSIN1*

1 *3+280. *PSIN**2*PSINZ**Z-840. *PSIN**2*PSIN3*ZETA2-28. *PSIN**2*PS

1 INS+10980. *PSIN**2*ZETAZ*ZETA4+3360.*PSIN**2*ZETA2**3+6720. *PSIN

$1 * * 2 * Z E T A 6-560$. *PSIN**3*PSIN1*PSINE+112Q. *PSIN**3*PSINE*ZETA2+56.

1 *PSIN**3*PSIN4-840.*PSIN**4*PSIN1*ZETA2+210. *PSIN**4*PSINI**2-70

1 . *PSIN**4*PSIN3+840.*PSIN**4*ZETA2**2+840.*PSIN**4*ZETA4+5E. *PSI

$1 N * * 5 * P S I N E-28$. *PSIN**6*PSIN1+5E. *PSIN**6*ZETA2+PSIN**B-28R. *PSIN

1 1*PSINE**2+84Q. *PSIN1*PSIN3*ZETAE+28. *PSIN1*PSIN5*10080. *PSIN1*Z

1 ETA2*ZETA4-3360. *PSIN1 *ZETA2**3-6720. *PSIN1*ZETA6-210. *PSIN1**2*

1 PSIN3+Z520. *PSIN1**2*ZミTA2**2+2520. *PSIN1**Z*ZETA4-840.*PSIN1**3

1 *ZETAE+105. *PSIN1**4+56. *PSIN2*PSIN4+560.*PSIN2**2*ZETA2-840.*PS

1 IN3*ZETAZ**2ं-840. *PSIN3*ZETA4+35.*PSIN3**E-56.*PSIN5*ZETAZ-PSIN7

$1+13440$. *ZETAE*ZETA6+1808Q.*ZETAZ**Z*ZETA4+1680.*ZETA2**4+504Q.*Z

1 ETA4**E'+10(1)

Note: $\operatorname{CPSI}(l+1)=\Psi_{\ell, \mathrm{n}} \quad \operatorname{ZETAC}=\zeta(l) \quad \operatorname{PSIN}=\psi(\mathrm{n}) \quad \operatorname{GAMIN}=1 . / \Gamma(\mathrm{n}) \quad \operatorname{PSIN} \ell=\psi^{\ell}(\mathrm{n})$ 
TABLE 2

Coefficients of the polynomials $P_{1 j}(\log x)$

\begin{tabular}{c|cr}
$j$ & coefficient $u_{j, 0}$ & (numerical) \\
\hline-1 & 1 & \\
0 & 0 & 0 \\
1 & $\pi^{2} / 12$ & .822467033424113 \\
2 & $-\zeta(3) / 3$ & -.400685634386531 \\
3 & $\pi^{4} / 160$ & .608806818962515 \\
4 & $-\zeta(3) \pi^{2} / 36-\zeta(5) / 5$ & -.536936276078223 \\
5 & $\zeta(3)^{2} / 18+61 \pi^{6} / 120960$ & .565102041778833 \\
6 & $-\zeta(3) \pi^{4} / 480-\zeta(5) \pi^{2} / 60-\zeta(7) / 7$ & -.558557822173266 \\
7 & $\zeta(3)^{2} \pi^{2} / 216+\zeta(3) \zeta(5) / 15+1261 \pi^{8} / 29030400$ & .561274982966818 \\
\hline
\end{tabular}

also $P_{1 j}=\sum_{k=1}^{j+1} u_{j, k} v^{k}$ where $v=\log \left(e^{\gamma} x\right)$ and $u_{j, k}=-u_{j-1, k-1} / k$

example: $P_{13}(v)=\zeta(3) v / 3+\pi^{2} v^{2} / 24+v^{4} / 24$

TABLE 3.1

$E_{1}^{1}(X)=P(X) / Q(X) *(X-X 11)+P 11(\operatorname{LOG}(X)$

$X 11=.91446 \quad 486597277$

IN THE RANGE 0.000.LE.X.LE. 1.000

RATIONAL APPROXIMATION IS R $(3,4)$

WITH PRECISION $=14.46$ DIGITS

\begin{tabular}{|c|c|c|c|c|}
\hline POO & 6) & -.376341 & 57664 & 06520 \\
\hline P01 & 5) & -.635070 & 75949 & 96412 \\
\hline P02 & 4) & -.538768 & 20580 & 92452 \\
\hline P03 & 2) & -.584198 & 45557 & 93210 \\
\hline Q00 & 6) & .418437 & 62176 & 08333 \\
\hline $\begin{array}{l}\text { Q01 } \\
\text { Q02 }\end{array}$ & $\begin{array}{l}\text { 6) } \\
\text { 5) }\end{array}$ & $\begin{array}{r}.121793 \\
.132630\end{array}$ & $\begin{array}{l}37377 \\
73906\end{array}$ & $\begin{array}{l}23431 \\
04789\end{array}$ \\
\hline Q0 & 3) & .551375 & 17596 & 10943 \\
\hline Q04 & 1) & . 100000 & 00000 & 00000 \\
\hline
\end{tabular}

$E_{1}^{1}(X)=\operatorname{EXP}(-X) / X * \star 2 * P(Z) / Q(Z) ; Z=1 / X$

IN THE RANGE 0.000.LE.Z.LE. .100

RATIONAL APPROXIMATION IS $\mathrm{R}(4,6)$

WITH PRECISION= 15.13 DIGITS

\begin{tabular}{|c|c|c|c|c|}
\hline POO & $-2)$ & . 368896 & 16541 & 46809 \\
\hline P01 & $0)$ & .111317 & 82274 & 12989 \\
\hline P02 & 1) & .111573 & 42563 & 49138 \\
\hline $\mathrm{PO3}$ & 1) & .430645 & 72608 & 32509 \\
\hline P04 & 1) & .522913 & 13537 & 89389 \\
\hline Q00 & $-2)$ & .368896 & 16541 & 46811 \\
\hline Q01 & $0)$ & .122384 & 70770 & 37284 \\
\hline 002 & 1) & .144230 & 98012 & 73127 \\
\hline Q03 & 1) & .747160 & 29601 & 50458 \\
\hline Q04 & 2) & .168869 & 92689 & 15484 \\
\hline Q05 & 2) & .135627 & 18800 & 19350 \\
\hline Q06 & 1) & .100000 & 00000 & 00000 \\
\hline
\end{tabular}

FIT TO $E^{1}{ }_{1}(X)=\operatorname{EXP}(-X) \star P(X) / Q(X)$

IN THE RANGE 1.000.LE.X.LE.10.000

RATIONAL APPROXIMATION IS $R(6,8)$ WITH PRECISION= 14.09 DIGITS

\begin{tabular}{|c|c|c|c|c|c|}
\hline P00 & ( & 1) & -.338130 & 76971 & 20518 \\
\hline P01 & & $0)$ & .891028 & 00665 & 24613 \\
\hline P02 & & 3) & .211811 & 97726 & 44376 \\
\hline P03 & & 3) & .283328 & 58929 & 93606 \\
\hline P04 & & 3) & .120102 & 26074 & 96511 \\
\hline P05 & ( & 2) & .192587 & 99072 & 06423 \\
\hline P06 & ( & $0)$ & 999999. & 85034 & 28858 \\
\hline Q00 & ( & $0)$ & -.400917 & 37819 & 6801 \\
\hline Q01 & ( & 2) & -.106209 & 25463 & 66475 \\
\hline Q02 & ( & 2) & .419023 & 64057 & 58286 \\
\hline Q03 & ( & 3) & .569128 & 54287 & 46715 \\
\hline Q04 & ( & 3) & .964803 & 08155 & 39357 \\
\hline Q05 & & 3) & .616101 & 47030 & 825 \\
\hline Q06 & ( & 3) & .175879 & 22239 & 84149 \\
\hline Q07 & ( & 2) & .222587 & 85540 & 75955 \\
\hline Q08 & ( & 1) & .100000 & 00000 & 00000 \\
\hline
\end{tabular}


TABLE 3.2

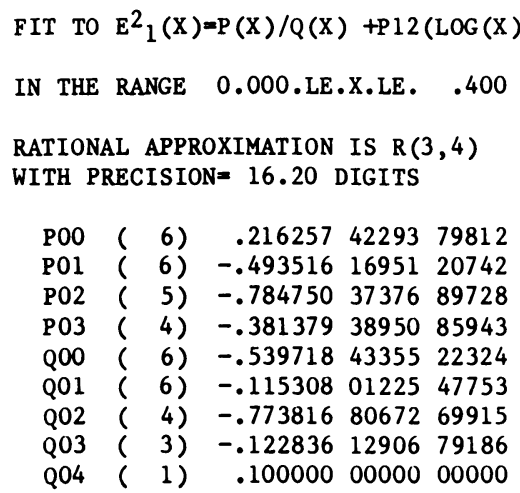

FIT TO $E^{2}{ }_{1}(X)=\operatorname{EXP}(-X) * P(X) / Q(X)$

IN THE RANGE 2.000.LE.X.LE.10.000

RATIONAL APPROXIMATION IS R $(5,8)$

WITH PRECISION $=15.55$ DIGITS

\begin{tabular}{|c|c|c|c|c|}
\hline POO & 3) & .340433 & 44179 & 16653 \\
\hline P01 & 3) & .876064 & 53575 & 85969 \\
\hline P02 & 3) & .676598 & 41845 & 71783 \\
\hline P03 & 3) & . 203399 & 00769 & 19242 \\
\hline P04 & 2) & .246893 & 21393 & 49231 \\
\hline P05 & $0)$ & 999999 . & 81273 & 60712 \\
\hline Q00 & 2) & .206456 & 40742 & 55514 \\
\hline Q01 & 4) & .120509 & 38588 & 4781 \\
\hline Q02 & 4) & .511338 & 37482 & 96754 \\
\hline Q03 & 4) & .778642 & 56924 & 73543 \\
\hline Q04 & 4) & .547543 & 10587 & 36117 \\
\hline Q05 & 4) & .194264 & 79236 & 68901 \\
\hline Q06 & 3) & .352535 & 91968 & 35532 \\
\hline Q07 & 2) & . 306893 & 01010 & 78642 \\
\hline Q08 & 1) & .100000 & 00000 & 00000 \\
\hline
\end{tabular}

FIT TO $E^{2}{ }_{1}(X)=\operatorname{EXP}(-X) * P(X) / Q(X)$

IN THE RANGE .400. LE.X.LE. 2.000

RATIONAL APPROXIMATION IS $\mathrm{R}(5,8)$

WITH PRECISION= 14.90 DIGITS

\begin{tabular}{|c|c|c|c|c|}
\hline P00 & 0) & .534274 & 07496 & 24268 \\
\hline P01 & 2) & .172848 & 04128 & 51346 \\
\hline P02 & 2) & .602765 & 67841 & 44 \\
\hline P03 & 2) & .582331 & 73035 & 43360 \\
\hline P04 & 2) & .147552 & 63525 & 88969 \\
\hline P05 & $0)$ & 999989. & 73572 & 67234 \\
\hline $\mathrm{Q} 00$ & $-1)$ & 170899. & 18403 & 89456 \\
\hline Q01 & 1) & .295254 & 33350 & 91985 \\
\hline Q02 & 2) & .651380 & 89668 & 07230 \\
\hline Q03 & 3) & . 315340 & 67127 & 43816 \\
\hline Q04 & 3) & .573546 & 38415 & 89567 \\
\hline Q05 & 3) & .444897 & 80147 & 56000 \\
\hline Q06 & 3) & .147788 & 79716 & 87537 \\
\hline Q07 & 2) & .207544 & 71393 & 35389 \\
\hline 08 & 1) & .100000 & 00000 & 00000 \\
\hline
\end{tabular}

$E^{2}{ }_{1}(X)=\operatorname{EXP}(-X) / X * * 3 * P(Z) / Q(Z) ; Z=1 / X$

IN THE RANGE 0.000 .LE.Z.LE. .100

RATIONAL APPROXIMATION IS R $(5,5)$

WITH PRECISION $=13.58$ DIGITS

$\begin{array}{llrll}\text { P00 } & (-3) & .325912 & 28045 & 69083 \\ \text { P01 } & (-2) & .976173 & 27349 & 42300 \\ \text { P02 } & (-1) & .894008 & 30387 & 59118 \\ \text { P03 } & (-1) & .249087 & 11994 & 57106 \\ \text { P04 } & (-1) & .139742 & 26502 & 81342 \\ \text { P05 } & (-2) & -.401189 & 07964 & 43870 \\ \text { Q00 } & (-3) & .325912 & 28045 & 69169 \\ \text { Q01 } & (-1) & .117172 & 06417 & 64891 \\ \text { Q02 } & (-1) & .148297 & 13910 & 05923 \\ \text { Q03 } & (10) & .802097 & 98704 & 24226 \\ \text { Q04 } & (1) & .174325 & 30019 & 86266 \\ \text { Q05 } & (1) & .100000 & 00000 & 00000\end{array}$

In the cases $j=5$ and 6, multiple-precision (48 digit) arithmetic [7] was employed within the master functions to overcome large losses in accuracy near the upper part of the range, and again, the lowest $(k+l)$ approximation with more than 14 digit accuracy was chosen for inclusion in Table 3 . In some cases, it was beneficial to split this range into two to obtain more efficient approximations.

3c. Large $x$. It proved fruitless to approximate $E_{1}^{j}(x)$ with the asymptotic formula (2.27) in the range $10 \leqslant x$. Accordingly, $(k, l)$ minimax fits $R_{j}(1 / x)$ were procured such that

$$
E_{1}^{j}(x)=\exp (-x) x^{-j-1} R_{j}(1 / x)
$$


TABLE 3.3

FIT TO $E^{3}{ }_{1}(X)=P(X) / Q(X)+P 13(\operatorname{LOG}(X))$

IN THE RANGE 0.000.LE.X.LE. .600

RATIONAL APPROXIMATION IS $\mathrm{R}(3,4)$ WITH PRECISION $=15.65$ DIGITS

\begin{tabular}{|c|c|c|c|c|}
\hline$P 00$ & 6) & -.839003 & 82605 & 47848 \\
\hline P01 & 7) & .119755 & 65275 & 64264 \\
\hline P02 & 6) & .242909 & 62547 & 68230 \\
\hline P03 & 5) & . 108775 & 12253 & 31120 \\
\hline Q00 & 7) & -.137811 & 17423 & .510 \\
\hline Q01 & 6) & -.296572 & 26101 & 03168 \\
\hline Q02 & 5) & -.174055 & 92800 & 54023 \\
\hline Q03 & 3) & -.157386 & 94906 & 03826 \\
\hline 04 & 1) & .100000 & 00000 & 00000 \\
\hline
\end{tabular}

FIT TO $E^{3}{ }_{1}(X)=\operatorname{EXP}(-X) \star P(X) / Q(X)$

IN THE RANGE .600. LE.X:LE .10 .000

RATIONAL APPROXIMATION IS $\mathrm{R}(5,9)$

WITH PRECISION= 13.37 DIGITS

\begin{tabular}{|c|c|c|c|c|}
\hline 00 & 2) & .157208 & 97422 & 57519 \\
\hline$? 01$ & 3) & .128368 & 95938 & 74056 \\
\hline 02 & 3) & . 270646 & 30105 & 87470 \\
\hline 3 & 3) & .148490 & 63549 & 0547 \\
\hline & 2) & .228734 & 95971 & \\
\hline 75 & 0) & .999998 & 89216 & 9484 \\
\hline 0 & $0)$ & . 320385 & 15630 & $x$ \\
\hline & 2) & .675468 & 89806 & 766 \\
\hline & 3) & .861412 & 12489 & 90 \\
\hline & 4) & .372041 & 91499 & 309 \\
\hline & 4) & .677596 & 67313 & \\
\hline & 4) & .552376 & 35994 & 4951 \\
\hline & 4) & .213346 & 91178 & 7990 \\
\hline & 3) & . 392231 & 03684 & 4513 \\
\hline & 2) & .328733 & 75166 & 0400 \\
\hline & 1) & .100000 & 00000 & 0000 \\
\hline
\end{tabular}

$$
\begin{aligned}
& E_{1}^{3}(X)=\operatorname{EXP}(-X) / X * \star 4 * P(Z) / Q(Z) ; Z=1 / X \\
& \text { IN THE RANGE 0.000.LE.Z.LE. } .100 \\
& \text { RATIONAL APPROXIMATION IS } \mathrm{R}(3,8) \\
& \text { WITH PRECISION }=14.31 \text { DIGITS } \\
& \begin{array}{lllll}
\text { P00 } & (-2) & -.174019 & 20356 & 94571 \\
\text { P01 } & (-1) & -.709402 & 14529 & 05107 \\
\text { P02 } & (-0) & -.905606 & 10531 & 27851 \\
\text { P03 } & (1) & -. .359745 & 25082 & 90533 \\
\text { Q00 } & (-2) & -.174019 & 20356 & 94563 \\
\text { Q01 } & (-1) & -. .883421 & 34886 & 03280 \\
\text { Q02 } & (1) & -.164111 & 11311 & 14874 \\
\text { Q03 } & (2) & -.137785 & 23506 & 45778 \\
\text { Q04 } & (2) & -.514428 & 97426 & 13443 \\
\text { Q04 } & (2) & -.514428 & 97426 & 13443 \\
\text { Q05 } & (2) & -.685703 & 73818 & 80260 \\
\text { Q06 } & (2) & -.162601 & 49416 & 93719 \\
\text { Q07 } & (2) & -.957694 & 76113 & 15230 \\
\text { Q08 } & (1) & .100000 & 00000 & 00000
\end{array}
\end{aligned}
$$

where $0 \leqslant 1 / x \leqslant 0.1$. To evaluate the master functions, Eq. (2.12) was transformed into the integral form

$$
E_{1}^{j}(x)=\exp (-x) x^{-j-1} \int_{0}^{\infty} d t \exp (-t)(1+t / x)^{-j-1} R_{j-1}\left(\frac{1}{t+x}\right),
$$

where $R_{j-1}$ was a 19 digit rational minimax fit to $x^{j} \exp (x) E_{1}^{j-1}(x)$ according to Eq. (3.6) obtained especially for that purpose. The integration was a 400-point Gauss-Laguerre calculation in double-precision arithmetic [5]. To begin the bootstrap, high-precision fits to $E_{1}(x)$ due to Cody and Thacher [9] in this range were employed. The fit in the Walsh table with smallest $(k+l)$ and greatest accuracy over 14 s.d. was chosen, and is reported in Table 3. 
TABLE 3.4

FIT TO $E^{4}{ }_{1}(X)=P(X) / Q(X)+P 14(\operatorname{LOG}(X))$

IN THE RANGE 0.000 .LE.X.LE. .500

RATIONAL APPROXIMATION IS R $(3,3)$ WITH PRECISION $=15.13$ DIGITS

\begin{tabular}{|c|c|c|c|c|}
\hline POO & 6) & .921856 & 63967 & 05455 \\
\hline P01 & 7) & .164159 & 41458 & 60650 \\
\hline P02 & 6) & -.112522 & 80878 & 61094 \\
\hline P03 & 3) & -.607131 & 03056 & 26315 \\
\hline Q00 & 7) & -.171688 & 27675 & 48834 \\
\hline Q01 & 6) & -.140218 & 91431 & 54977 \\
\hline Q02 & 4) & -.161995 & 44008 & 14657 \\
\hline$J 3$ & 1) & .100000 & 00000 & 00000 \\
\hline
\end{tabular}

FIT TO $E^{4}{ }_{1}(X)=\operatorname{EXP}(-X) * P(X) / Q(X)$

IN THE RANGE 2.000.LE.X.LE. 10.000

RATIONAL APPROXIMATION IS $\mathrm{R}(4,8)$ WITH PRECISION $=13.93$ DIGITS

\begin{tabular}{|c|c|c|c|c|}
\hline 00 & 2) & .353537 & 04817 & 300 \\
\hline P01 & 2) & .293820 & 63659 & 486 \\
\hline P02 & 2) & .145436 & 89922 & 68919 \\
\hline P03 & $0)$ & 999939 . & 71607 & 90340 \\
\hline P04 & $-6)$ & .520751 & 56693 & 51248 \\
\hline Q00 & 1) & -.223458 & 69506 & 60041 \\
\hline Q01 & 3) & .204802 & 30991 & 30036 \\
\hline Q02 & 4) & .183199. & 07073 & 94430 \\
\hline Q03 & 4) & .333915 & 80415 & 91970 \\
\hline Q04 & 4) & . 276686 & 23525 & 75097 \\
\hline Q05 & 4) & .128585 & 73754 & 40614 \\
\hline Q06 & 3) & .297624 & 44755 & 03964 \\
\hline Q07 & & .295401 & 74603 & 13003 \\
\hline & & 100000 & 00000 & 00000 \\
\hline
\end{tabular}

FIT TO $E^{4}{ }_{1}(X)=\operatorname{EXP}(-X) * P(X) / Q(X)$

IN THE RANGE .500.LE.X.LE. 2.000

RATIONAL APPROXIMATION IS $R(6,6)$

WITH PRECISION= 13.75 DIGITS

$\begin{array}{llrll}\text { P00 } & (-1) & .190007 & 72856 & 75969 \\ \text { P01 } & (-0) & .145255 & 03101 & 02014 \\ \text { P02 } & (-1) & .812092 & 21046 & 70916 \\ \text { P03 } & (-2) & -.725683 & 52768 & 34330 \\ \text { P04 } & (-3) & .559368 & 64008 & 87053 \\ \text { P05 } & (-4) & -.321053 & 49168 & 10291 \\ \text { P06 } & (-6) & .996850 & 83917 & 42953 \\ \text { Q00 } & (-4) & .383398 & 00706 & 66617 \\ \text { Q01 } & (-1) & .950926 & 03238 & 66574 \\ \text { Q02 } & (-1) & .175435 & 39262 & 79244 \\ \text { Q03 } & (1) & .827856 & 65799 & 05180 \\ \text { Q04 } & (1) & .117836 & 83566 & 14576 \\ \text { Q05 } & (1) & .609668 & 97953 & 70546 \\ \text { Q06 } & (1) & .100000 & 00000 & 00000\end{array}$

$E^{4}{ }_{1}(X)=E X P(-X) / X * * 5 * P(Z) / Q(Z) ; Z=1 / X$

IN THE RANGE 0.000.LE.Z.LE. .100

RATIONAL APPROXIMATION IS $\mathrm{R}(5,7)$

WITH PRECISION= 14.84 DIGITS

$\begin{array}{llrll}\text { P00 } & (-5) & .395133 & 17174 & 71982 \\ \text { P01 } & (-3) & .204661 & 41023 & 99156 \\ \text { P02 } & (-2) & .346003 & 84617 & 74784 \\ \text { P03 } & (-1) & .204274 & 58543 & 62413 \\ \text { P04 } & (-1) & .211759 & 92902 & 12236 \\ \text { P05 } & (-2) & -.228430 & 28733 & 96219 \\ \text { Q00 } & (-5) & .395133 & 17174 & 71976 \\ \text { Q01 } & (-3) & .263931 & 38600 & 20275 \\ \text { Q02 } & (-2) & .672752 & 62012 & 18175 \\ \text { Q03 } & (-1) & .828969 & 69188 & 14699 \\ \text { Q04 } & (-0) & .515915 & 51447 & 03679 \\ \text { Q05 } & (1) & .155462 & 70935 & 57096 \\ \text { Q06 } & (1) & .206789 & 80159 & 55259 \\ \text { Q07 } & (1) & .100000 & 00000 & 00000\end{array}$

In all three ranges, the function $E_{1}^{j}(x)$ computed by rational $(k, l)$ fits in single precision was compared with the multiple-precision master routines for 20,000 pseudo-random values of $x$ and $1 / x$. The largest fractional error noted was $1.1 \times$ $10^{-13}$.

Finally, the functions $E_{-n}^{j}(x)$ were calculated for $1 \leqslant j \leqslant 7,0 \leqslant n \leqslant 7$ and pseudo-random values of $x$ and $1 / x$ according to Eq. (2.22). Comparisons were made with the same functions calculated via Eq. (2.3) using the single-precision routine DCADRE [10]. No significant discrepancies were found at the relative error level of $1 \times 10^{-12}$, and it was observed that rational minimax methods require $\sim 150$ times less computation time than does DCADRE. For the case $j=0$, a pre-existant library function based on [9] was used, although better methods are known [3]. Agreement with Gussman's tabulated values [11] was total. 
TABLE 3.5

FIT TO $E^{5}{ }_{1}(X)=P(X) / Q(X)+P 15(\operatorname{LOG}(X))$ IN THE RANGE 0.000 .LE.X.LE. .300 RATIONAL APPROXIMATION IS $\mathrm{R}(3,2)$ WITH PRECISION $=15.30$ DIGITS

$\begin{array}{llrrr}\text { P00 } & (3) & .838743 & 38519 & 74414 \\ \text { P01 } & (4) & -.142760 & 40312 & 21125 \\ \text { P02 } & (4) & -.880503 & 85483 & 46258 \\ \text { P03 } & (4) & -.556431 & 96858 & 95740 \\ \text { Q00 } & (4) & .148423 & 35068 & 49911 \\ \text { Q01 } & (3) & .100211 & 06179 & 41411 \\ \text { Q02 } & (3) & .100000 & 00000 & 00000\end{array}$

FIT TO $E^{5}{ }_{1}(X)=\operatorname{EXP}(-X) * P(X) / Q(X)$

IN THE RANGE 2.000.LE.X.LE. 10.000

RATIONAL APPROXIMATION IS $R(4,9)$

WITH PRECISION $=14.90$ DIGITS

\begin{tabular}{|c|c|c|c|c|}
\hline POO & 2) & .185231 & 13891 & 15313 \\
\hline P01 & 2) & . 169071 & 89408 & 0932 \\
\hline P02 & 2) & .163822 & 45019 & 47477 \\
\hline P03 & 0) & 999973 & 45809 & 7364 \\
\hline P04 & $-6)$ & .178967 & 24703 & 8604 \\
\hline Q00 & $0)$ & -.332104 & 62172 & 4037 \\
\hline Q01 & 3) & .127144 & 45160 & 39896 \\
\hline Q02 & 4) & .223717 & 64184 & \\
\hline Q03 & 4) & .632007 & 63231 & 3991 \\
\hline Q04 & 4) & .803518 & 43255 & 5934 \\
\hline Q05 & 4) & .632932 & 10036 & 443 \\
\hline Q06 & 4) & .259400 & 04237 & 2968 \\
\hline Q07 & 3) & .479992 & 93424 & 76704 \\
\hline Q08 & 2) & . 373802 & 66825 & 57554 \\
\hline Q09 & 1) & .100000 & 00000 & 0000 \\
\hline
\end{tabular}

FIT TO $E^{5}(X)=E X P(-X) * P(X) / Q(X)$

IN THE RANGE .300.LE.X.LE. 2.000

RATIONAL APPROXIMATION IS $R(5,10)$ WITH PRECISION $=15.44$ DIGITS

\begin{tabular}{|c|c|c|c|c|}
\hline & $-3)$ & .500522 & & \\
\hline & $0)$ & 9471 & 65122 & \\
\hline & 1) & 234 & & \\
\hline P03 & 1) & . 379468 & 68767 & 0673 \\
\hline P04 & 0) & . 991769 & 17992 & 22935 \\
\hline P05 & $-3)$ & .144274 & 11935 & 15928 \\
\hline Q00 & $-4)$ & .322199 & 80730 & 277 \\
\hline Q01 & $-3)$ & .967191 & 28566 & 38765 \\
\hline Q02 & 1) & .114091 & 98728 & 01536 \\
\hline Q03 & 2) & .364550 & 65926 & 78242 \\
\hline Q04 & 3) & .320690 & 08398 & 60978 \\
\hline Q05 & 3) & .977517 & 80646 & 43698 \\
\hline Q06 & 4) & .126115 & 41159 & 04314 \\
\hline Q07 & 3) & .736669 & 39202 & 94918 \\
\hline Q08 & & .2019 & 35278 & 47405 \\
\hline Q09 & & .245286 & 16349 & 83681 \\
\hline & & .10 & 0000 & 00 \\
\hline
\end{tabular}

$E^{5}{ }_{1}(X)=E X P(-X) / X * \star 6 * P(Z) / Q(Z) ; Z=1 / X$

IN THE RANGE 0.000.LE.Z.LE. .100

RATIONAL APPROXIMATION IS $\mathrm{R}(5,7)$

WITH PRECISION $=14.05$ DIGITS

$\begin{array}{llrll}\text { P00 } & (-6) & .854783 & 10464 & 47894 \\ \text { P01 } & (-4) & .624139 & 17717 & 77879 \\ \text { P02 } & (-2) & .138649 & 42989 & 29153 \\ \text { P03 } & (-2) & .948336 & 88932 & 79712 \\ \text { P04 } & (-3) & -.423100 & 66195 & 40255 \\ \text { P05 } & (-4) & -.370718 & 93388 & 82177 \\ \text { Q00 } & (-6) & .854783 & 10464 & 47817 \\ \text { Q01 } & (-4) & .803643 & 62915 & 37229 \\ \text { Q02 } & (-2) & .279890 & 57603 & 97328 \\ \text { Q03 } & (-1) & .462603 & 61191 & 05448 \\ \text { Q04 } & (-1) & .380244 & 88250 & 95635 \\ \text { Q05 } & (1) & .147127 & 35379 & 71468 \\ \text { Q06 } & (1) & .229031 & 45317 & 90874 \\ \text { Q07 } & (1) & .100000 & 00000 & 00000\end{array}$


TABLE 3.6

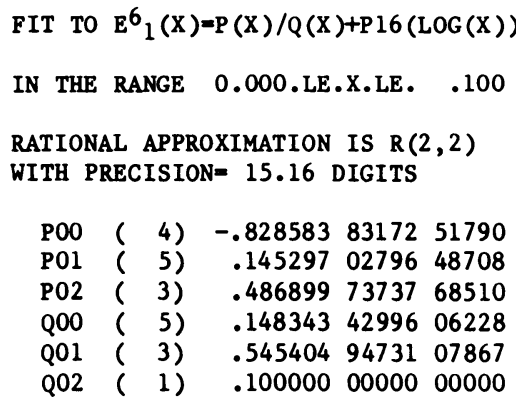

FIT TO $E^{6}{ }_{1}(X)=\operatorname{EXP}(-X) * P(X) / Q(X)$

IN THE RANGE 2.000.LE.X.LE. 10.000

RATIONAL APPROXIMATION IS $\mathrm{R}(7,7)$

WITH PRECISION= 14.29 DIGITS

\begin{tabular}{|c|c|c|c|c|}
\hline P00 & 1) & .109840 & 75266 & 90525 \\
\hline P01 & $-2)$ & -.628986 & 68217 & 39343 \\
\hline P02 & $-3)$ & .352279 & 00224 & 82096 \\
\hline P03 & $-4)$ & -.166654 & 15521 & 17304 \\
\hline P04 & $-6)$ & .631863 & 11472 & 37745 \\
\hline P05 & $-7)$ & -.177898 & 75053 & 81043 \\
\hline P06 & $-9)$ & .327229 & 37007 & 14598 \\
\hline P07 & $(-11)$ & -.292513 & 40388 & 70733 \\
\hline Q00 & $(0)$ & .174130 & 46755 & 89622 \\
\hline Q01 & 1) & .789054 & 90506 & 24093 \\
\hline 02 & 3) & .294284 & 05239 & 39408 \\
\hline Q03 & 3) & .980479 & 98971 & 36415 \\
\hline Q04 & 3) & .851988 & 71787 & 21233 \\
\hline 05 & 3) & .259397 & 92046 & 62330 \\
\hline Q06 & 2) & .293770 & 63084 & 51261 \\
\hline Q07 & 1) & .100000 & 00000 & 00000 \\
\hline
\end{tabular}

FIT TO $E^{6}{ }_{1}(X)=E X P(-X) \star P(X) / Q(X)$

IN THE RANGE .100.LE.X.LE. 2.000

RATIONAL APPROXIMATION IS $\mathrm{R}(6,12)$ WITH PRECISION $=14.72$ DIGITS

\begin{tabular}{|c|c|c|c|c|}
\hline $\begin{array}{l}\text { P00 } \\
\text { P01 }\end{array}$ & $\begin{array}{l}-3) \\
-1)\end{array}$ & $\begin{array}{r}.271478 \\
.195102\end{array}$ & $\begin{array}{l}83310 \\
35413\end{array}$ & $\begin{array}{l}87788 \\
21396\end{array}$ \\
\hline P02 & $0)$ & .321565 & 23084 & 02674 \\
\hline P03 & 1) & .138382 & 85866 & 93074 \\
\hline P04 & 1) & .205248 & 36427 & 90331 \\
\hline P05 & $0)$ & .989908 & 76978 & 43590 \\
\hline P06 & $-3)$ & .167302 & 21625 & 00549 \\
\hline Q00 & $-7)$ & -.771598 & 59706 & 76959 \\
\hline Q01 & $-2)$ & .168613 & 64843 & 91203 \\
\hline Q02 & $0)$ & .219806 & 16317 & 34924 \\
\hline Q03 & 1) & .810987 & 78546 & 02619 \\
\hline Q04 & 3) & .114098 & 17140 & 23387 \\
\hline Q05 & 3) & .688229 & 02344 & 19855 \\
\hline Q06 & 4) & .205074 & 91268 & 54280 \\
\hline Q07 & 4) & . 325821 & 03449 & 06946 \\
\hline Q08 & 4) & .282942 & 94513 & 61645 \\
\hline Q09 & 4) & .130505 & 73635 & 81354 \\
\hline Q10 & 3) & .296709 & 78069 & 04683 \\
\hline Q11 & 2) & .296971 & 60052 & 15741 \\
\hline Q12 & 1) & .100000 & 00000 & 00000 \\
\hline
\end{tabular}

$E^{6}{ }_{1}(X)=\operatorname{EXP}(-X) / X * \star 7 * P(Z) / Q(Z) ; Z=1 / X$

IN THE RANGE 0.000.LE.Z.LE. .100

RATIONAL APPROXIMATION IS $\mathrm{R}(6,7)$

WITH PRECISION $=14.98$ DIGITS

$\begin{array}{lrrrr}\text { P00 } & (-6) & .117928 & 10446 & 90701 \\ \text { P01 } & (-5) & .887532 & 43497 & 71136 \\ \text { P02 } & (-3) & .210604 & 43465 & 37132 \\ \text { P03 } & (-2) & .154902 & 34850 & 49091 \\ \text { P04 } & (-3) & -.627848 & 92801 & 53793 \\ \text { P05 } & (-3) & .202298 & 30636 & 59744 \\ \text { P06 } & (-4) & -.409048 & 68342 & 68896 \\ \text { Q00 } & (-6) & .117928 & 10446 & 90700 \\ \text { Q01 } & (-4) & .121773 & 11274 & 90613 \\ \text { Q02 } & (-3) & .487180 & 40530 & 95863 \\ \text { Q03 } & (-2) & .965568 & 34655 & 76481 \\ \text { Q04 } & (-0) & .100200 & 50733 & 04699 \\ \text { Q05 } & (10) & .527459 & 47929 & 77240 \\ \text { Q06 } & (1) & .125439 & 02841 & 63380 \\ \text { Q07 } & (1) & .100000 & 00000 & 00000\end{array}$


Acknowledgments. The author is grateful to J. Blair for many helpful discussions, to S. Jurgilas for aid with the REMES2 runs, and to R. Davis for demonstrating that Eq. (2.23) can be evaluated in FORTRAN for arbitrary values of $j$. The referee pointed out the existence of [26] from which the further reference to [11] was found.

\section{Appendix A}

\section{A Short Table of Integrals}

From the $G$-function representation (2.7) and integrals listed in [21], it is possible to generate a table of very general integrals, which contain many useful results as special cases, all of which are believed to be new. A short list follows.

$$
\begin{aligned}
& \int_{0}^{\infty} d t t^{\gamma} E_{\mu}^{j}(\alpha t) E_{\nu}^{k}(\beta t) \\
& \quad=\beta^{-\gamma-1} G_{j+k+3, j+k+3}^{j+2, k+2}
\end{aligned}\left(\begin{array}{l}
\left.\frac{\alpha}{\beta} \mid \begin{array}{l}
-\gamma, 1-\nu-\gamma, \ldots, 1-\nu-\gamma ; \mu, \ldots, \mu \\
0, \mu-1, \ldots, \mu-1 ;-\nu-\gamma, \ldots,-\nu-\gamma
\end{array}\right) .
\end{array}\right.
$$

Special case $(j=-1)$

$$
\begin{aligned}
\int_{0}^{\infty} & t^{\gamma} e^{-\alpha t} E_{\nu}^{k}(\beta t) d t \\
& =\beta^{-\gamma-1} \sum_{l=0}^{\infty} \frac{\Gamma(1+\gamma+l)}{\Gamma(1+l)}\left(\frac{1}{\nu+\gamma+l}\right)^{k+1}\left(\frac{-\alpha}{\beta}\right)^{l} \text { if }|\alpha| \leqslant|\beta| .
\end{aligned}
$$

Special case $(k=\gamma=0, \nu=\alpha=\beta=1, \mu=m$; [20], [24])

$$
\int_{0}^{\infty} E_{1}(t) E_{m}^{j}(t) d t
$$

$$
=\frac{(-1)^{j} j !}{m^{j+1}}\left\{\frac{1}{2} \sum_{l=0}^{j} \frac{(-1)^{l}}{l !}\left(\frac{m}{2}\right)^{\prime}\left[\psi^{\prime}\left(\frac{m+1}{2}\right)-\psi^{\prime}\left(\frac{m}{2}\right)\right]+\log 2\right\} .
$$

$$
\int_{0}^{\infty} t^{\gamma} E_{\nu}^{k}(\beta t) d t=\beta^{-\gamma-1} \Gamma(1+\gamma)(\nu+\gamma)^{-k-1} \text {. }
$$

$$
\begin{aligned}
& \int_{0}^{\infty} t^{\gamma-1}(t+\beta)^{-\sigma} E_{\nu}^{j}(z t) d t \\
& =\frac{\beta^{\gamma-\sigma}}{\Gamma(\sigma)} G_{j+2, j+3}^{j+3,1}\left(\left.\beta z\right|_{\sigma-\gamma, 0, \nu-1, \ldots, \nu-1 ;} ^{1-\gamma ; \nu, \ldots, \nu} .\right.
\end{aligned}
$$

Special case $(\gamma=1, \sigma=1+\nu, j=0)$

$$
\int_{0}^{\infty}(t+\beta)^{-\nu-1} E_{\nu}(z t) d t=\beta^{-\nu} \exp (\beta z) E_{\nu}(\beta z) / \nu .
$$

Special case $(\sigma=2, \gamma=2-\nu, j=0)$

$$
\begin{aligned}
& \int_{0}^{\infty} t^{1-\nu}(t+\beta)^{-2} E_{\nu}(z t) d t=\exp (\beta z) z^{\nu-1} \Gamma(2-\nu) E_{2-\nu}(\beta z) \\
& \int_{0}^{\infty}{ }_{2} F_{1}\left(\begin{array}{c}
\alpha, \beta \\
\gamma
\end{array} \mid-w t\right) t^{\delta} E_{\nu}^{j}(z t) d t \\
& =\frac{z^{-\delta}}{w} \frac{\Gamma(\gamma)}{\Gamma(\alpha) \Gamma(\beta)} G_{j+3, j+4}^{j+4,1}\left(\frac{z}{w} \mid \begin{array}{l}
0 ; \gamma-1, \nu+\delta, \ldots, \nu+\delta \\
\delta, \nu+\delta-1, \ldots, \nu+\delta-1, \alpha-1, \beta-1 ;
\end{array}\right)
\end{aligned}
$$


Special case $(\alpha=\beta=\delta=1, \gamma=2)$

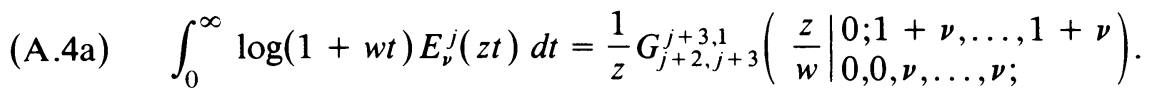

Special case $\left(\beta=\alpha-\frac{1}{2}, \gamma=2 \alpha, \delta=\alpha-1-\nu\right)$

(A.4b)

$$
\int_{0}^{\infty}[1+\sqrt{1+w t}]^{1-2 \alpha} t^{\alpha-1-\nu} E_{\nu}^{j}(z t) d t
$$

$$
=\frac{z^{\nu+1-\alpha}\left(\alpha-\frac{1}{2}\right)}{w \sqrt{\pi}} G_{j+2, j+3}^{j+3,1}\left(\frac{z}{w} \mid \begin{array}{l}
0 ; 2 \alpha-1, \alpha-1, \ldots, \alpha-1 \\
\alpha-1-\nu, \alpha-\frac{3}{2}, \alpha-2, \ldots, \alpha-2 ;
\end{array}\right),
$$

(A.5) $\int_{0}^{\infty} \cos (\alpha t) E_{\nu}^{j}(\beta t) d t$

$$
\begin{aligned}
& =\frac{2^{-j-1}}{\alpha} G_{j+2, j+2}^{j+2,1}\left(\frac{\beta^{2}}{\alpha^{2}} \mid \begin{array}{l}
\frac{1}{2} ;(\nu+1) / 2, \ldots,(\nu+1) / 2 \\
\frac{1}{2},(\nu-1) / 2, \ldots,(\nu-1) / 2
\end{array}\right) \\
& =\frac{2^{-j-1}}{\beta} \sum_{l=0}^{\infty}\left(\frac{1}{\nu / 2+l}\right)^{j+1}\left(\frac{-\alpha^{2}}{\beta^{2}}\right)^{l} \quad \text { if }|\alpha| \leqslant|\beta|,
\end{aligned}
$$

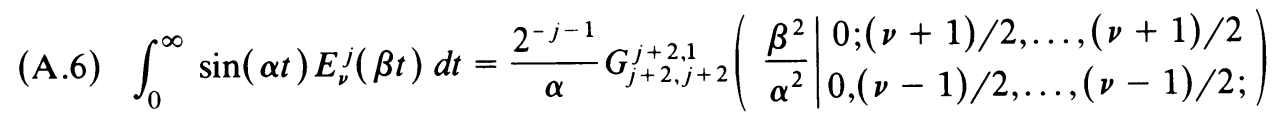

(A.6a) $\quad=\frac{2^{-j-1} \alpha}{\beta^{2}} \sum_{l=0}^{\infty}\left(\frac{1}{(1+\nu) / 2+l}\right)^{j+1}\left(\frac{-\alpha^{2}}{\beta^{2}}\right)^{l}$ if $|\alpha| \leqslant|\beta|$.

(A.7)

$$
\begin{aligned}
& \int_{1}^{\infty} t^{-\alpha}(t-1)^{\alpha-\beta-1} E_{s}^{j}(z t) d t \\
& \quad=\Gamma(\alpha-\beta) G_{j+2, j+3}^{j+3,0}\left(z \mid \begin{array}{l}
; s, \ldots, s, \alpha \\
0, \beta, s-1, \ldots, s-1 ;
\end{array}\right) .
\end{aligned}
$$

Special case $(s=-n, \alpha=1-l, \beta=l)$

$$
\int_{1}^{\infty} t^{t-1} E_{-n}^{j}(z t) d t
$$

$(\mathrm{A} .7 \mathrm{a})=\frac{\Gamma(n+1)}{z^{n+1}}\left[\sum_{k=0}^{n-j} \frac{z^{k}}{k !} \xi_{k, n}^{j} E_{n+2-k-l}(z)+E_{n+1-l}(z) \sum_{m=1}^{j} \xi_{0, n}^{j-m}\left(\frac{1}{n-l}\right)^{m}\right.$

$$
\begin{array}{r}
\left.-\sum_{m=1}^{j} \sum_{i=0}^{m-1}(n-l)^{i-m} \xi_{0, n}^{j-m} E_{1}^{i}(z)\right] \\
\text { if } l \neq n, n+1, \\
\int_{1}^{\infty} t^{n-1} E_{-n}^{j}(z t) d t
\end{array}
$$

$$
=\frac{\Gamma(n+1)}{z^{n+1}}\left[\sum_{k=0}^{n-j} \frac{z^{k}}{k !} \xi_{k, n}^{j} E_{2-k}(z)+\sum_{m=1}^{j} \xi_{0, n}^{m-1} E_{1}^{j-m+1}(z)\right]
$$

For the special case $l=n+1$, see Eq. (2.12). 
Applied Mathematics Branch

Atomic Energy of Canada, Limited

Chalk River, Ontario, Canada KOJ $1 \mathrm{~J} 0$

1. M. Abramowitz \& I. Stegun, Handbook of Mathematical Functions, Chapter 5, U. S. National Bureau of Standards, Washington, DC, 1964.

2. I. K. Abu-Shumays, Transcendental Functions Generalizing the Exponential Integrals, Northwestern University (unpublished) report COO-2280-6. 1973.

3. D. E. Amos, "Computation of exponential integrals," ACM Trans. Math. Software, v. 6, 1980, pp. 365-377.

4. L. BERG, "On the estimation of the remainder term in the asymptotic expansion of the exponential integral," Computing, v. 18, 1977, pp. 361-363.

5. B. S. BERGER, Tables of Zeros and Weights for Gauss-Laguerre Quadrature to $24 \mathrm{~S}$ for $n=400,500$, 600, Dept. of Mechanical Engineering, Univ. of Maryland, College Park, MD. (Unpublished report.)

6. W. F. BREIG \& A. L. CROSBIE, "Numerical computation of a generalized exponential integral function," Math. Comp., v. 28, 1974, pp. 575-579.

7. R. P. BRENT, "A FORTRAN multiple-precision arithmetic package," ACM Trans. Math. Software, v. 4, 1978, pp. 57-70; ibid. pp. 71-81.

8. S. Chandrasekhar, Radiative Transfer, Dover, New York, 1960.

9. W. J. CODY \& H. C. ThaChER, JR., "Rational Chebyshev approximations for the exponential integral $E_{1}(x)$." Math. Comp., v. 22, 1968, pp. 641-649.

10. DCADRE, IMSL Library, 6th Floor, GNB Bldg., 7500 Bellaire Blvd., Houston, TX.

11. E. A. Gussman, "Modification to the weighting function method for the calculation of Fraunhofer lines in solar and stellar spectra," Z. Astrophys., v. 65, 1967, pp. 456-497.

12. H. C. VAN DE HulST, "Scattering in a planetary atmosphere," Astrophys. J., v. 107, 1948, pp. $220-246$

13. H. C. VAn DE Hulst, Multiple Light Scattering, Vol. 1, Academic Press, New York, 1980.

14. D. R. Jeng, E. J. LeE \& K. J. DE WITT, "Exponential integral kernels appearing in the radiative heat flux," Indian J. Tech., v. 13, 1975, pp. 72-75.

15. J. H. Johnson \& J. M. Blair, REMES2: A FORTRAN Programme to Calculate Rational Minimax Approximations to a Given Function, Atomic Energy of Canada Ltd., Report AECL-4210, 1973.

16. C. Kaplan, On a Generalization of the Exponential Integral, Aerospace Research Lab. Report ARL-69-0120, 1969; On Some Functions Related to the Exponential Integrals, Aerospace Research Lab. Report ARL-70-0097, 1970; Asymptotic and Series Expansion of the Generalized Exponential Integrals, Air Force Office of Scientific Research Interim Report AFOSR-TR-72-2147, 1972.

17. J. LE CAINE, $A$ Table of Integrals Involving the functions $E_{n}(x)$, National Research Council of Canada Report NRC-1553, 1945, Section 1.6.

18. Y. L. Luke, The Special Functions and Their Approximations, Academic Press, New York, 1969.

19. A. S. Meligy \& E. M. El Gazzy, "On the function $E_{n}(z)$," Proc. Cambridge Philos. Soc., v. 59. 1963, pp. 735-737.

20. M. S. Milgram, “Approximate solutions to the half-space integral transport equation near a plane boundary," Canad. J. Phys., v. 58, 1980, pp. 1291-1310.

21. M. S. Milgram, "Some properties of the solution to the integral transport equation in semi-infinite plane geometry," Atomkernenergie, v. 38, 1981, pp. 99-106.

22. M. S. Milgram, "Solution of the integral transport equation across a place boundary," Proc. ANS / ENS International Topical Meeting on Advances in Mathematical Methods for the Solution of Nuclear Engineering Problems, Munich, FDR, (1981), pp. 207-217.

23. W. Neuhaus \& S. Schottlander, "The development of Aireys converging factors of the exponential integral to a representation with remainder term," Computing, v. 15, 1975, pp. 41-52.

24. M. A. Sharaf, "On the $\Lambda$-transform of the exponential integrals," Astrophys. and Space Sci., v. 60, 1979, pp. 199-212.

25. R. R. Sharma \& B. Zohuri, "A general method for an accurate evaluation of exponential integrals $E_{1}(x), x>0$," J. Comput. Phys., v. 25, 1977, pp. 199-204.

26. A. Stankiewicz, "The generalized integro-exponential functions," Acta Univ. Wratislav., No. 188. 1973. pp. $11-42$.

27. I. A. Stegun \& R. Zucker, "Automatic computing methods for special functions. Part II," J. Res. Nat. Bur. Standards, v. 78B, 1974, pp. 199-218.

28. H. Strubbe, "Development of the SCHOONSCHIP program," Comput. Phys. Comm., v. 18, 1979 , pp. $1-5$.

29. R. TERRAS, "The determination of incomplete gamma functions through analytic integration," $J$. Comput. Phys., v. 31, 1979, pp. 146-151. 